\title{
An overview of $\Lambda$-type operations on quasi-symmetric functions
}

\author{
K. Bertet *, D. Krob ${ }^{\dagger}, \quad$ M. Morvan ${ }^{\ddagger}$, \\ J.-C. Novelli §, H.D. Phan ^ and J.-Y. Thibon ॥ \\ Dedicated to the memory of Professor A. I. Kostrikin
}

\begin{abstract}
We present an overview of $\Lambda$-type operations on the algebra of quasi-symmetric functions. Nous présentons un survol de l'ensemble des propriétés de type $\Lambda$-anneau de l'algèbre des fonctions quasi-symétriques.
\end{abstract}

\section{Introduction}

The algebra of noncommutative symmetric functions Sym, introduced in [5], is the free associative algebra (over some field of characteristic 0 ) generated by an infinite sequence $\left(S_{n}\right)_{n \geq 1}$ of noncommuting indeterminates (corresponding to the complete symmetric functions), endowed with some extra structure imitated from the usual algebra of commutative symmetric functions.

Noncommutative symmetric functions were intensively studied in a series of seminal papers $([5,9,4,10,11])$. It is shown there that noncommutative symmetric functions provide a new point of view on several important algebraic objects such as the free Lie algebra $($ see $[5,4])$, Solomon's descent algebra (see $[5,9,4]$ ), the 0-Hecke algebra (see $[10,11]$ ) or crystal limits of classical quantum groups of type $A$ (see $[10,11]$ ) for which they give natural descriptions of their representation theory.

It happens that the graded dual of Sym can be identified with the algebra QSym of quasisymmetric functions that was introduced by Gessel in [6]. This other aspect of the theory of noncommutative symmetric functions is clearly also very important, but it was however not really very well explored up to now, especially if one thinks that QSym can also be seen as a natural extension of ordinary commutative symmetric functions.

In this direction, an important question would be to know whether there exists a good generalization of the notion of $\Lambda$-ring for which the algebra of quasi-symmetric functions would

\footnotetext{
* L3I - Université de La Rochelle - Avenue Michel Crépeau - 17042 La Rochelle Cedex 1 - France kbertet@univ-lr.fr

${ }^{\dagger}$ LIAFA - Université Paris 7 - 2, place Jussieu - 75251 Paris Cedex 05 - France - dk@liafa.jussieu.fr

‡ LIAFA - Université Paris 7 - 2, place Jussieu - 75251 Paris Cedex 05 - France - morvan@liafa.jussieu.fr

$\S$ Corresponding author: LIFL - Batiment M3 - Cité Scientifique - Université Lille I - 59655 Villeneuve D’Ascq Cedex - France - novelli@lifl.fr

ฯ LIAFA - Université Paris 7 - 2, place Jussieu - 75251 Paris Cedex 05 - France - phan@liafa.jussieu.fr

"| IGM - Université de Marne-la-Vallée - 2, rue de la Butte Verte - 93166 Noisy-le-Grand Cedex - France jyt@univ-mlv.fr
} 
be an universal object, exactly as it is the case for the usual algebra of commutative symmetric functions with respect to the usual structure of $\Lambda$-ring (see [8]). This question is however still open, the main difficulty being the fact that it seems rather difficult to find a representation theoretic interpretation of the natural notion of plethysm of quasi-symmetric functions, introduced by Malvenuto and Reutenauer (see[14]).

In this paper, we are therefore trying to explore the $\Lambda$-ring type operations that can be defined on quasi-symmetric functions, as it was already done in the context of noncommutative symmetric functions (see [9]). We present first a survey of all known definitions of $\Lambda$-ring type operations on QSym. We then focus on the most complicated operation of this kind, i.e., the socalled plethysm of quasi-symmetric functions, for which we propose a number of new algorithms, based on lattice theory, for efficiently computing it.

\section{Definitions and notations}

\subsection{Partially ordered sets}

Let $P=\left(V,<_{P}\right)$ be a poset over some set $V$. Two elements $a, b \in V$ are said to be comparable in $P$ if one has either $a<b$, or $a>b$. Two elements that are not comparable, are said to be incomparable. A poset is then called an antichain if all its elements are pairwise incomparable.

We now need to introduce a generic operation on posets, called series-composition and denoted by $*$. Let hence $P=\left(U,<_{P}\right)$ and $Q=\left(V,<_{Q}\right)$ be two posets with disjoint ground sets $U$ and $V$. We denote then by $R=P * Q$ the series-composition of $P$ and $Q$ which is the poset constructed on $W=U \cup V$ which ordering relation $<_{R}$ is defined by setting $x<_{R} y$ if one has either $x<_{P} y$ with $x, y \in U$, either $x<_{Q} y$ with $x, y \in V$ or $x \in U$ and $y \in V$.

A poset $R$ is then said to be a weak order if it is obtained by series-composition of a finite number of antichains, i.e., if $R$ is of the form

$$
R=A_{1} * A_{2} * \ldots * A_{k}
$$

where each $A_{i}$ is an antichain. An element $x$ in such a weak order $R$ is said to be of height $r$ if it belongs to $A_{r}$. The number $k$ of antichains involved in the previous construction of $R$ is called the height of $R$.

A poset $Q=\left(U,<_{Q}\right)$ is finally said to be an extension of a poset $P=\left(U,<_{P}\right)$ constructed on the same ground set $U$ iff one has $u<_{Q} v$ as soon as one has $u<_{P} v$. A weak-order extension of $P$ is then an extension of $P$ which is also a weak order. In the same way, a weak-order linear quotient is a total order constructed on a quotient of a weak order extension of a given poset.

Let now $P=\left([1, n],<_{P}\right)$ be a poset over the interval $[1, n]$ of the set $\mathbb{N}$ of all positive integers. A linear extension of such a poset $P$ is then just a permutation of $\mathfrak{S}_{n}$ that extends $P$ to a total order. In other words, a permutation $\sigma \in \mathfrak{S}_{n}$ is a linear extension of $P$ iff one has

$$
i<_{P} j \Longrightarrow \sigma^{-1}(i)<\sigma^{-1}(j)
$$

for every $i, j \in[1, n]$. The set of all linear extensions of $P$ is denoted by $L(P)$.

We can now introduce a construction on posets over initial intervals of the set $\mathbb{N}^{*}$ of all strictly positive integers. Let therefore $P=\left([1, p],<_{P}\right)$ and $Q=\left([1, q],<_{Q}\right)$ be two posets respectively over $[1, p]$ and $[1, q]$. We denote then by $P \rightarrow Q$ (resp. by $P \leftarrow Q$ ) the poset constructed over $[1, p+q]$ whose order $<$ is the smallest order over $[1, p+q]$ such that 


$$
p<p+1 \text { (resp. } p>p+1) \text { and }\left\{\begin{array}{rll}
i<j & \Longleftrightarrow i<_{P} j & \text { if } i, j \in[1, p], \\
i<j & \Longleftrightarrow i-p<_{Q} j-p & \text { if } i, j \in[p+1, p+q] .
\end{array}\right.
$$

In other words, the Hasse diagram of $P \rightarrow Q$ (resp. $P \leftarrow Q$ ) is obtained as follows: re-index first the support of $Q$ by translating it over $[p+1, p+q]$, take then together the Hasse diagrams of $P$ and $Q$, add finally an arrow that relates the last element $p$ of the support of $P$ and the first element $p+1$ of the support of $Q$ oriented from $p$ to $p+1$ (resp. from $p+1$ to $p$ ). To illustrate our construction, let us consider the posets $T_{12}$ and $T_{2}$ given by the following Hasse diagrams.

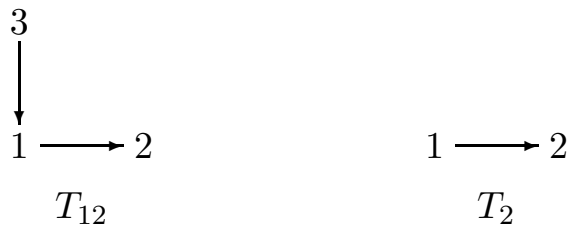

The Hasse diagram of the posets $T_{12} \leftarrow T_{2}$ and $T_{12} \rightarrow T_{2}$ are then given below.

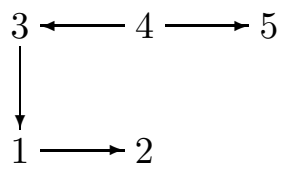

$T_{12} \leftarrow T_{2}$

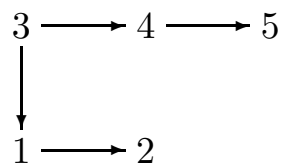

$T_{12} \rightarrow T_{2}$

\subsection{Compositions}

A composition of $n$ is a sequence $I=\left(i_{1}, \ldots, i_{r}\right)$ of strictly positive integers whose sum is equal to $n$. The length $\ell(I)$ of the composition $I$ is then just the number $r$ of integers involved in such a sequence. One usually represents a composition $I$ by a ribbon diagram of shape $I$, i.e., by a skew Young tableau of ribbon shape $I$. For instance, the ribbon diagram associated with the composition $I=(3,2,1,4)$ is given below.

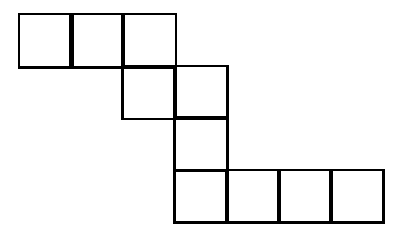

Let $I=\left(i_{1}, \ldots, i_{r}\right)$ be a composition. Its mirror image is then the composition denoted by $\bar{I}$ which is defined by reading from right to left the entries of $I$, i.e., by setting $\bar{I}=\left(i_{n}, \ldots, i_{1}\right)$. Using the graphical representation of a composition, one can define the conjugate composition of $I$, denoted by $I^{\sim}$, that is the composition obtained by enumerating from right to left the numbers of cells of the columns of the ribbon diagram of $I$. One can read for instance on the above picture that $(3,2,1,4)^{\sim}=(1,1,1,3,2,1,1)$.

We also need to introduce two classical operations on compositions. Let $I=\left(i_{1}, \ldots, i_{r}\right)$ and $J=\left(j_{1}, \ldots, j_{s}\right)$ be two compositions. We then define the compositions $I \cdot J$ and $I \triangleleft J$ by setting respectively $I \cdot J=\left(i_{1}, \ldots, i_{r}, j_{1}, \ldots, j_{s}\right)$ and $I \triangleleft J=\left(i_{1}, \ldots, i_{r-1}, i_{r}+j_{1}, j_{2}, \ldots, j_{s}\right)$. Notice that 
these two operations graphically correspond to the two ways of gluing the ribbon diagram of $I$ at the left of the ribbon diagram of $J$ as shown below when $I=(2,1)$ and $J=(1,2)$.

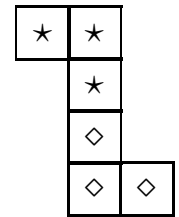

$(2,1) \cdot(1,2)$

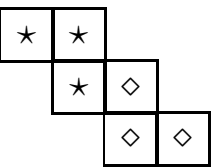

$(2,1) \triangleleft(1,2)$

One can associate with every composition $I=\left(i_{1}, \ldots, i_{r}\right)$ of $n$ the subset $D(I)$ of $[1, n-1]$ defined by setting $D(I)=\left\{i_{1}, i_{1}+i_{2}, \ldots, i_{1}+\ldots+i_{r-1}\right\}$. One then defines the refinement ordering, denoted $\prec$, on the set of all compositions of the same integer $n$ by setting $I \prec J$ iff $D(I) \subset D(J)$. One has for example $(3,2,6) \prec(2,1,2,3,1,2)$.

We are now in position to present a classical way for associating with every permutation $\sigma$ of $\mathfrak{S}_{n}$ a composition $C(\sigma)$ of $n$. Let us first recall that the descent set of $\sigma \in \mathfrak{S}_{n}$ is the subset of $[1, n-1]$, denoted by $\mathcal{D}(\sigma)$, formed of all integers $1 \leq i \leq n-1$ such that $\sigma(i)>\sigma(i+1)$. The composition $C(\sigma)$ is then by definition the unique composition of $n$ such that $D(C(\sigma))=\mathcal{D}(\sigma)$. One has for instance $C(245316)=(3,1,2)$ as one can see on the following picture.

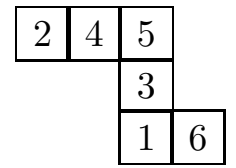

\section{Quasi-symmetric functions}

Quasi-symmetric functions were introduced by Gessel in [6]. Their Hopf algebra structure was studied by Gessel, Malvenuto and Reutenauer (see $[6,13]$ ). Their relationship with noncommutative symmetric functions was investigated by Duchamp, Gelfand, Krob, Lascoux, Leclerc, Malvenuto, Retakh, Reutenauer and Thibon (see [13, 5, 9, 4, 10, 11]).

\subsection{Definition and classical bases}

Let $X=\left\{x_{1}<x_{2}<\cdots<x_{n}<\cdots\right\}$ be an infinite totally ordered set of commutative indeterminates. A function $f$ of $\mathbb{C}[X]$ is said to be a quasi-symmetric function if, for each composition $K=\left(k_{1}, \ldots, k_{m}\right)$, all monomials $x_{i_{1}}^{k_{1}} x_{i_{2}}^{k_{2}} \ldots x_{i_{m}}^{k_{m}}$ with $i_{1}<i_{2}<\ldots<i_{m}$ have the same coefficient in $f$. The quasi-symmetric functions form a subalgebra of $\mathbb{C}[X]$ denoted by $Q S y m$. This algebra is naturally graded by the graduation $\left(Q S y m_{n}\right)_{n \geq 0}$ inherited from $\mathbb{C}[X]$.

One associates with every composition $I=\left(i_{1}, \ldots, i_{m}\right)$ the quasi-monomial function denoted by $M_{I}$ by setting

$$
M_{I}=\sum_{j_{1}<\ldots<j_{m}} x_{j_{1}}^{i_{1}} \ldots x_{j_{m}}^{i_{m}}
$$

By construction, the family of quasi-monomial functions is a natural basis of QSym. It follows in particular from this fact that the dimension of the $\mathbb{C}$-vector space $Q S y m_{n}$ is $2^{n-1}$. Another important basis of $Q S y m$ is formed by the quasi-ribbon functions $\left(F_{I}\right)$ defined by setting

$$
F_{I}=\sum_{I \prec J} M_{J}
$$


for every composition $I$.

One can also give a combinatorial description of these functions that shows that they play the role of Schur functions in our context. Let again $I$ be a composition. Let us first recall that a quasi-ribbon of shape $I$ is a ribbon diagram of shape $I$ where every box contains a letter of $X$ in such a way that:

1. the letters in every row are increasing from left to right;

2. the letters in every column are strictly increasing from top to bottom.

One can associate with every quasi-ribbon the commutative monomial obtained by taking the product of all its letters. The quasi-ribbon function $F_{I}$ is then the sum of the commutative monomials associated with all quasi-ribbons of shape $I$ (see $[6,10]$ ).

Example 3.1 Let $X=\{x<y<z\}$ and $I=(2,1)$. The quasi-ribbon function $F_{21}(X)$ is equal to

$$
\begin{array}{|l|l|l|}
\hline x & x \\
\hline y
\end{array}+\begin{array}{|l|l|l|}
\hline x & x \\
\hline
\end{array}+\begin{array}{|l|l|l|}
\hline x & y \\
\hline
\end{array},
$$

i.e., $F_{21}(X)=x^{2} y+x^{2} z+y^{2} z+x y z=M_{21}(X)+M_{111}(X)$.

It is also interesting to give explicitly the graded generating functions of the two bases of QSym that we introduced.

Proposition 3.2 Let $X$ be a totally ordered alphabet. Then one has

$$
\sum_{I} t^{\ell(I)} M_{I}=\frac{\prod_{x \in X}(1+(t-1) x)}{\prod_{x \in X}(1-x)} .
$$

Proof - It suffices to notice that one has

$$
\sum_{I} t^{\ell(I)} M_{I}=\sum_{\lambda} t^{\ell(\lambda)} \psi_{\lambda}
$$

where $\psi_{\lambda}$ denotes the monomial symmetric function indexed by the partition $\lambda$. The result then becomes a simple exercise on symmetric functions that is left to the reader.

Corollary 3.3 Let $X$ be a totally ordered alphabet. Then one has

$$
\sum_{I} t^{\ell(I)} F_{I}=\frac{1}{1+t}+\frac{t}{1+t} \frac{\prod_{x \in X}(1+t x)}{\prod_{x \in X}(1-x)} .
$$

Proof - According to [5], one can write

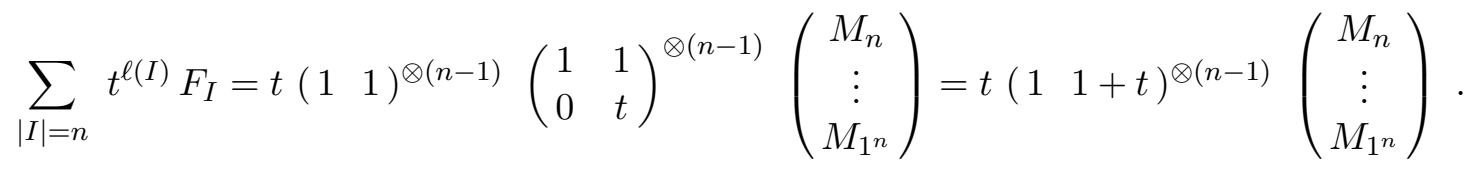


In other words, one has

$$
\sum_{|I|=n} t^{\ell(I)} F_{I}=\frac{t}{1+t}\left(\sum_{|I|=n}(1+t)^{\ell(I)} M_{I}\right),
$$

for every $n \geq 1$. The desired formula now immediately follows from this last relation and from Proposition 3.2.

\subsection{Quasi-symmetric functions associated with posets}

We recall here a construction due to Gessel (see [6]) that allows to associate a quasi-symmetric function with every finite poset.

Definition 3.4 (Stanley; $[16])$ Let $P=\left([1, n],<_{P}\right)$ be a poset over the interval $[1, n]$ of $\mathbb{N}$. A $P$-partition is then a function $f$ from $[1, n]$ into $X$ satisfying the two conditions:

1. $i<_{P} j$ implies $f(i) \leq f(j)$;

2. $i<_{P} j$ and $i>j$ implies $f(i)<f(j)$.

Let again $P=\left([1, n],<_{P}\right)$ be a poset over $[1, n]$. We denote then by $C_{P}$ the function which is the sum of all commutative monomials $f(1) f(2) \ldots f(n)$ where $f$ runs over the $P$-partitions corresponding to $P$. It appears that $C_{P}$ is in fact a quasi-symmetric function.

Proposition 3.5 (Gessel; $[6])$ Let $P=\left([1, n],<_{P}\right)$ be a poset over $[1, n]$. The function $C_{P}$ is then a quasi-symmetric function. Moreover one has:

$$
C_{P}=\sum_{f P-\text { partition }} \prod_{i=1}^{n} f(i)=\sum_{\sigma \in L(P)} F_{C(\sigma)} .
$$

Example 3.6 Let us consider the poset $P=\left(\{1,2,3,4\},<_{P}\right)$ defined by

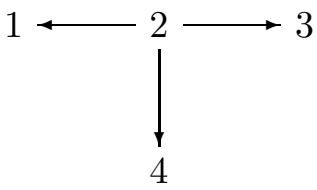

Let $X=\{x<y<z\}$. The $P$-partitions associated with $P$ exactly are the functions $f$ from $[1,4]$ into $X$ such that $f(2)<f(1), f(2) \leq f(3), f(2) \leq f(4)$. On the other hand, one has $L(P)=\{(2134),(2143),(2314),(2341),(2413),(2431)\}$. Thus Proposition 3.5 shows that

$$
C_{P}(X)=F_{13}+F_{121}+2 F_{22}+F_{31}+F_{211} .
$$

We associate with every composition $I$ of $n$ a poset over $[1, n]$, denoted by $T_{I}$, which is defined as follows. Let us first consider the skew Young tableau $\Theta_{I}$ obtained by filling the rows of the ribbon diagram of ribbon shape $I$ from left to right, beginning with the bottom-most row and ending with the top-most row with the integers from 1 to $n$. When $I=(2,3,1)$, one obtains for instance the following skew Young tableau.

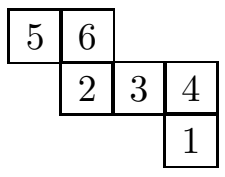


The poset $T_{I}=\left([1, n],<_{I}\right)$ is then the smallest poset such that $i<_{I} j$ if either $i$ is to the left of $j$ in its row, or $i$ is to the bottom of $j$ in its column. Continuing the previous example leads us therefore to the poset $T_{231}$ given below.

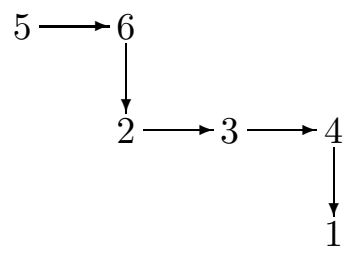

It appears that the consideration of these posets allows to simplify the usual description of the product of two quasi-ribbon functions (see [6,4]) into a formula that may be seen as an analog of the multiplication formula of noncommutative ribbon functions (see [5]).

Proposition 3.7 Let I, J be two compositions. Then one has

$$
F_{I} F_{J}=C_{T_{I} \leftarrow T_{J}}+C_{T_{I} \rightarrow T_{J}} .
$$

Example 3.8 Proposition 3.7 shows that the product $F_{22} F_{1}$ can be for instance expressed as the sum of the quasi-symmetric functions associated with the two following posets.
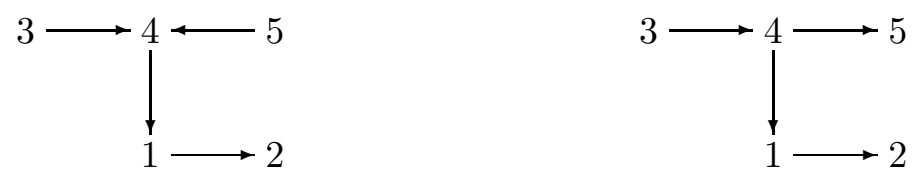

Using Proposition 3.5, we immediately get

$$
F_{22} F_{1}=F_{122}+F_{212}+F_{221}+F_{23}+F_{32},
$$

since it is easily checked that the linear extensions of the two previous posets are exactly the permutations listed below.
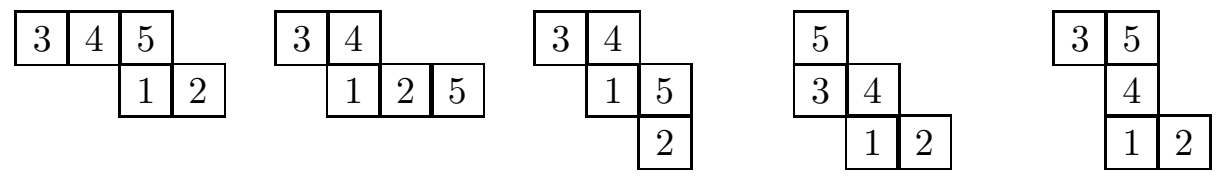

Note 3.9 There does not seem to exist such a simple expression for expressing the product of two quasi-monomials functions. Using the natural pairing between QSym and the algebra of noncommutative symmetric functions (see [5, 13]), one can however easily prove that one has for every compositions $I$ and $J$ :

$$
M_{I} M_{J}=\sum_{\substack{K=C+D \\ C \in I \boldsymbol{\Psi} 0^{*}, D \in J \boldsymbol{m} 0^{*}}} M_{K},
$$

where $K$ stands for a composition and where $C, D$ are vectors of $\mathbb{N}^{\ell(K)}$. To illustrate this last result, let us for instance consider $I=(2,1)$ and $J=(1,3)$. The compositions $K$ that may appear in identity (7) are then listed below: 


$$
\begin{gathered}
(2,1)+(1,3)=(3,4), \\
(0,2,1)+(1,3,0)=(1,5,1),(0,2,1)+(1,0,3)=(1,2,4),(2,0,1)+(0,1,3)=(2,1,4), \\
(2,0,1)+(1,3,0)=(3,3,1),(2,1,0)+(0,1,3)=(2,2,3),(2,1,0)+(1,0,3)=(3,1,3), \\
(0,0,2,1)+(1,3,0,0)=(1,3,2,1),(0,2,0,1)+(1,0,3,1)=(1,2,3,1), \\
(0,2,1,0)+(1,0,0,3)=(1,2,1,3),(2,1,0,0)+(0,0,1,3)=(2,1,1,3), \\
(2,0,1,0)+(0,1,0,3)=(2,1,1,3),(2,0,0,1)+(0,1,3,0)=(2,1,3,1) .
\end{gathered}
$$

Hence it follows that we have

$$
\begin{aligned}
M_{21} M_{13}= & M_{34}+M_{151}+M_{124}+M_{214}+M_{331}+M_{223}+M_{313} \\
& +M_{1321}+M_{1231}+M_{1213}+2 M_{2113}+M_{2131} .
\end{aligned}
$$

\section{$4 \quad \Lambda$-ring type operations on QSym}

The object of this section is to present an overview of the different $\Lambda$-ring type operations (see $[12,8]$ for instance) that can be defined on QSym. Thus we first recall how one usually defines the sum and the product of two totally ordered alphabets in the context of quasisymmetric functions. We focus then in a second time on the notion of plethysm of quasisymmetric functions as introduced by Malvenuto and Reutenauer in [14].

\subsection{Sum and product of ordered alphabets}

Let $X$ and $Y$ be two totally ordered alphabets. We denote then by $X \hat{+} Y$ the alphabet which is obtained by taking the disjoint union of $X$ and $Y$ ordered by setting $x<y$ for every $x \in X$ and $y \in Y$. Using the natural pairing between QSym and the algebra of Non-commutative symmetric functions (see [5]), one can easily obtain the following explicit formulas that express the quasi-monomials and the quasi-ribbon functions of the alphabet $X \hat{+} Y$ in terms of the same quasi-symmetric functions for the alphabets $X$ and $Y$.

Proposition 4.1 Let I be a composition. Then one has

$$
\begin{gathered}
M_{I}(X \hat{+} Y)=\sum_{P \cdot Q=I} M_{P}(X) M_{Q}(Y) \\
F_{I}(X \hat{+} Y)=\sum_{P \cdot Q=I} F_{P}(X) F_{Q}(Y)+\sum_{P \triangleleft Q=I} F_{P}(X) F_{Q}(Y) .
\end{gathered}
$$

Example 4.2 The decompositions of a composition $I$ involved in formula (9) can be easily read on its graphic representation. It suffices indeed to cut in all possible ways the ribbon diagram associated with $I$ into two disjointed parts in order to obtain all decompositions of the form $I=P \cdot Q$ and $I=P \triangleleft Q$. Let us consider for instance $I=(1,2,1)$. One can then obtain $F_{I}(X \hat{+} Y)$ by considering the following decomposition : 


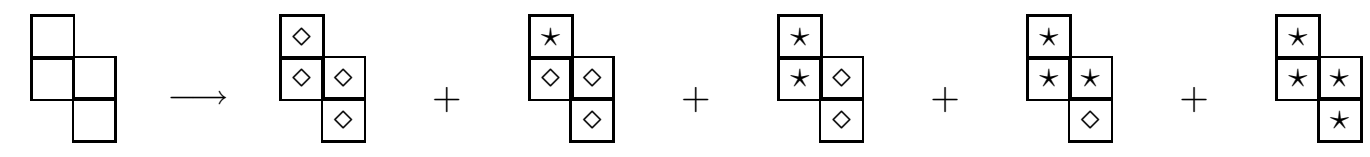

from which one gets

$$
F_{121}(X \hat{+} Y)=F_{121}(Y)+F_{1}(X) F_{21}(Y)+F_{11}(X) F_{11}(Y)+F_{12}(X) F_{1}(Y)+F_{121}(X)
$$

Let again $X$ be a totally ordered alphabet. The opposite alphabet $-X$ is then the totally ordered alphabet defined by setting for every composition $I$ :

$$
F_{I}(-X)=(-1)^{|I|} F_{I \sim(X)} .
$$

One can also express any quasi-monomial function of an opposite alphabet in terms of the quasi-monomial functions of the initial alphabet.

Proposition 4.3 (Malvenuto-Reutenauer; [13]) Let I be a composition. Then one has

$$
M_{I}(-X)=\sum_{I \succ J}(-1)^{\ell(J)} M_{\bar{J}}(X)
$$

Note 4.4 One can rephrase the previous definitions in terms of Hopf algebras. Using the identification $Q S y m \otimes Q S y m \simeq Q \operatorname{Sym}(X \hat{+} Y)$, one can indeed define a coproduct $\gamma$ on QSym by setting $\gamma(f)=f(X \hat{+} Y)$ for every quasi-symmetric function $f$. In the same way, one can also introduce the automorphism $\nu$ of QSym defined by setting $\nu(f)=f(-X)$ for every quasisymmetric function $f$. The algebra QSym equipped with its usual product - and with the coproduct $\gamma$ then has a structure of Hopf algebra with antipode $\nu$.

Let $X$ and $Y$ be again two totally ordered alphabets. One denotes then by $X \hat{\times} Y$ the alphabet obtained by totally ordering the set $X \times Y$ by lexicographic order. The following result gives an expression for the quasi-ribbon functions of the alphabet $X \hat{\times} Y$ in terms of the quasi-ribbon functions of the alphabets $X$ and $Y$.

Proposition 4.5 (Gessel; [6]) Let I be a composition and let $\pi$ be a permutation such that $C(\pi)=I$. Then one has:

$$
F_{I}(X \hat{\times} Y)=\sum_{\sigma \in \mathfrak{S}_{n}} F_{C(\sigma)}(X) F_{C\left(\pi \circ \sigma^{-1}\right)}(Y)
$$

Example 4.6 Let $I=(1,2)$ and let $\pi=213$ in $\mathfrak{S}_{3}$. Observe that one has $C(213)=(1,2)$. One can now construct the following table 


\begin{tabular}{|c|c|c|c|}
\hline$\sigma$ & $C(\sigma)$ & $\pi \circ \sigma^{-1}$ & $C\left(\pi \circ \sigma^{-1}\right)$ \\
\hline 123 & $(3)$ & 213 & $(1,2)$ \\
132 & $(2,1)$ & 231 & $(2,1)$ \\
213 & $(1,2)$ & 123 & $(3)$ \\
231 & $(2,1)$ & 321 & $(1,1,1)$ \\
312 & $(1,2)$ & 132 & $(2,1)$ \\
321 & $(1,1,1)$ & 312 & $(1,2)$ \\
\hline
\end{tabular}

from which one gets, according to Formula (11) :

$$
\begin{aligned}
F_{12}(X \hat{\times} Y)= & F_{3}(X) F_{12}(Y)+F_{21}(X) F_{21}(Y)+F_{12}(X) F_{3}(Y) \\
& +F_{21}(X) F_{111}(Y)+F_{12}(X) F_{21}(Y)+F_{111}(X) F_{12}(Y) .
\end{aligned}
$$

Note 4.7 When applied on symmetric functions, the different operations that we introduced for totally ordered alphabets reduce to the corresponding operations considered in the sense of the usual $\Lambda$-ring structure of the ring of symmetric functions (see [8, 12, 6, 5]).

\subsection{Plethysm of quasi-symmetric functions}

Let $X$ be a totally ordered alphabet and let $F$ and $G$ be two quasi-symmetric functions over $X$ with non negative integer coefficients. Following Malvenuto and Reutenauer (see [14]), one can define the plethysm of $F$ by $G$ as follows. Let us first decompose $G$ as a sum of commutative monomials totally ordered by lexicographic order, i.e.,

$$
G=\sum_{k \in K} m_{k}
$$

where some monomials are possibly equal (but distinguished by the total ordering of $K$ ). Elementary considerations then show that the function $F \circ G$ defined by setting

$$
F \circ G=F\left(\left\{m_{k}, k \in K\right\}\right)
$$

is a quasi-symmetric function over $X$ that we shall call plethysm of $F$ by $G$.

Example 4.8 Let $X=\{x<y<z\}$. Then one has

$$
2 M_{12}(x, y, z)=x y^{2}+x y^{2}+x z^{2}+x z^{2}+y z^{2}+y z^{2} .
$$

The plethysm $M_{12} \circ\left(2 M_{12}\right)$ over $X$ is then equal to

$$
\begin{gathered}
\left(M_{12} \circ\left(2 M_{12}\right)\right)(x, y, z)=\left(x y^{2}\right)\left(\left(x y^{2}\right)^{2}+\left(x z^{2}\right)^{2}+\left(x z^{2}\right)^{2}+\left(y z^{2}\right)^{2}+\left(y z^{2}\right)^{2}\right) \\
+\left(x y^{2}\right)\left(\left(x z^{2}\right)^{2}+\left(x z^{2}\right)^{2}+\left(y z^{2}\right)^{2}+\left(y z^{2}\right)^{2}\right)+\left(x z^{2}\right)\left(\left(x z^{2}\right)^{2}+\left(y z^{2}\right)^{2}+\left(y z^{2}\right)^{2}\right) \\
+\left(x z^{2}\right)^{2}\left(\left(y z^{2}\right)^{2}+\left(y z^{2}\right)^{2}\right)+\left(y z^{2}\right)^{2}\left(y z^{2}\right)^{2}
\end{gathered}
$$

which, as easily checked, reduces to

$$
\left(M_{12} \circ\left(2 M_{12}\right)\right)(x, y, z)=M_{36}(x, y, z)+4 M_{324}(x, y, z)+4 M_{144}(x, y, z)+4 M_{126}(x, y, z)
$$


The following proposition gives an important positiveness property of the plethysm of two quasi-ribbon functions.

Proposition 4.9 (Malvenuto, Reutenauer; [14]) Let I, J be two compositions. Then there exists a family $\left(p_{I, J}^{K}\right)$ of non negative integers such that

$$
\left(F_{I} \circ F_{J}\right)(X)=\sum_{K} p_{I, J}^{K} F_{K}(X) .
$$

Note 4.10 It is unfortunately not very clear whether this notion of plethysm should be put at the same level that the two other $\Lambda$-ring type operations introduced in the previous subsection. One indeed does not know for the moment any representation theoretical interpretation (in the line of $[10,11])$ of this plethystic operation on quasi-symmetric functions.

\section{$5 \quad$ Algorithms for computing plethysms}

The argument of Malvenuto and Reutenauer used to prove Proposition 4.9 is quite effective, but can be improved. The purpose of this section is to provide a number of simple algorithms based on posets properties for efficiently computing the plethysm of two quasi-monomials functions or the plethysm of two quasi-ribon functions on the corresponding bases of quasi-symmetric functions.

\subsection{Plethysm of quasi-monomial functions}

Let $X$ be a totally ordered infinite set and let $I, J$ be two compositions. We first address the problem of computing the plethysm of the quasi-monomial functions over the basis of quasimonomial functions. In other words, we will see how to compute the decomposition:

$$
M_{I}(X) \circ M_{J}(X)=\sum_{K \in \mathcal{K}} p_{I, J}^{K} M_{K}(X) .
$$

Let us now set $I=\left(i_{1}, \ldots, i_{n}\right)$ and $J=\left(j_{1}, \ldots, j_{m}\right)$. When $I=(i)$, note first that it is easy to check that one has:

$$
M_{(i)}(X) \circ M_{J}(X)=M_{i \times J}(X) .
$$

We will therefore assume that $n>1$ in all the sequel of our subsection. To compute the plethysm $M_{I}(X) \circ M_{J}(X)$, let us first write explicitly the definition of $M_{J}(X)$ :

$$
M_{J}(X)=\sum_{y_{1}<\cdots<y_{m} \in X} y_{1}^{j_{1}} \cdots y_{m}^{j_{m}}
$$

The commutative monomials that occur in the right hand-side of the previous expression can be totally ordered by lexicographic order. Thus, according to the definition of the plethysm of two quasi-symmetric functions given in Section 4.2, one gets:

$$
\begin{aligned}
M_{I}(X) \circ M_{J}(X) & =M_{I}\left(\left\{y_{1}^{j_{1}} \cdots y_{m}^{j_{m}}, y_{1}<\cdots<y_{m} \in X\right\}\right) \\
& =\sum_{y_{1}<\cdots<y_{m} \in X}\left(y_{1,1}^{j_{1}} \cdots y_{1, m}^{j_{m}}\right)^{i_{1}} \cdots\left(y_{n, 1}^{j_{1}} \cdots y_{n, m}^{j_{m}}\right)^{i_{n}} \\
& =\sum_{Y \in \mathcal{Y}} \prod_{\substack{k \in[1, n] \\
l \in[1, m]}} y_{k, l}^{i_{k} j_{l}}
\end{aligned}
$$


where the last above sum is taken over the set $\mathcal{Y}$ of all $n \times m$ matrices

$$
\left(\begin{array}{ccc}
y_{1,1} & \ldots & y_{1, m} \\
\vdots & \vdots & \vdots \\
y_{n, 1} & \ldots & y_{n, m}
\end{array}\right)
$$

whose entries stand in $X$ and which satisfy the two following conditions:

- Condition $\mathbf{H}$ : for every $k \in[1, n]$, one has $y_{k, 1}<\cdots<y_{k, m}$;

- Condition $\mathbf{V}$ : the row vectors of the matrix $Y$ are strictly increasing for the lexicographic order over $X^{m}$, i.e., one has

$$
\left(y_{1,1}, \ldots, y_{1, m}\right) \prec\left(y_{2,1}, \ldots, y_{2, m}\right) \prec \cdots \prec\left(y_{n, 1}, \ldots, y_{n, m}\right)
$$

where $\prec$ stands for the (strict) lexicographic order over $X^{m}$.

In order to describe all the matrices of the set $\mathcal{Y}$, we will decompose Condition $\mathbf{V}$ into a disjoint union of conditions of the same type. Observe first that each strict inequality between two adjacent rows

$$
\left(y_{k, 1}, \ldots, y_{k, m}\right) \prec\left(y_{k+1,1}, \ldots, y_{k+1, m}\right),
$$

is equivalent to the existence of an index $r_{k}$ for which rows $k$ and $k+1$ begin to differ, i.e., such that

$$
\begin{array}{ccccccc}
y_{k, 1} & \ldots & y_{k, r-1} & y_{k, r} & y_{k, r+1} & \ldots & y_{k, m} \\
= & \ldots & = & \wedge & & & \\
y_{k+1,1} & \ldots & y_{k+1, r-1} & y_{k+1, r} & y_{k+1, r+1} & \ldots & y_{k+1, m} .
\end{array}
$$

Conversely, let $t=\left(t_{1}, \ldots, t_{n-1}\right)$ be an element of the set $T$ of all vectors of $[1, m]^{n-1}$. Let $V(t)$ be the set of matrices of $\mathcal{M}_{n, m}(X)$ satisfying the condition $\mathbf{V}(\mathbf{t})$ :

- Condition $\mathbf{V}(\mathbf{t})$ : for every $k \in[1, n-1]$,

$$
\left\{\begin{array}{l}
\forall l \in\left[1, t_{k}-1\right], y_{l, k}=y_{l, k+1}, \\
y_{t_{k}, k}<y_{t_{k}, k+1} .
\end{array}\right.
$$

Then it is clear that a matrix satisfies Condition $\mathbf{V}$ if and only if there exist an element $t$ of $T$ such that this matrix satisfies Condition $\mathbf{V}(\mathbf{t})$. Moreover, if $t$ exists, it is unique. In other words, the set of matrices satisfying Condition $\mathbf{V}$ exactly is the disjoint union of the sets $\mathcal{V}(t)$ where $t$ runs over the set $T$.

For every $t \in T$, let us now define

$$
\Gamma_{t}(X)=\sum_{Y \in \mathcal{H} \cap \mathcal{V}(t)} \prod_{\substack{k \in[1, n] \\ l \in[1, m]}} y_{k, l}^{i_{k} j_{l}}
$$

where $\mathcal{H}$ stands for the set of all matrices of $\mathcal{M}_{n, m}(X)$ satisfying Condition $\mathbf{H}$. It is then easy to see that the plethysm of $M_{I}$ and $M_{J}$ can be rewritten as 


$$
M_{I}(X) \circ M_{J}(X)=\sum_{t \in T} \Gamma_{t}(X)
$$

Hence, to decompose the plethysm $M_{I}(X) \circ M_{J}(X)$ over the basis of quasi-monomials functions, it is enough to obtain such a decomposition for every $\Gamma_{t}(X)$ since one can easily prove that this function is a quasi-symmetric function. Let us therefore choose $t$ in $T$ and let $Y \in \mathcal{M}_{n, m}(X)$ satisfying both conditions $\mathbf{H}$ and $\mathbf{V}(\mathbf{t})$. One can then group together the entries of $Y$ in the different generic equivalence classes associated with the equality relation, i.e., in the different classes that consist of all the entries of $Y$ that can be proved equal, using Conditions $\mathbf{H}$ and $\mathrm{V}(\mathrm{t})$.

Example 5.1 Consider for instance the compositions $I=(3,5,7)$ and $J=(2,4,6,8)$ and the vector $t=(3,4)$. A matrix $Y \in \mathcal{M}_{3,4}(X)$ satisfying Conditions $\mathbf{H}$ and $\mathbf{V}(\mathbf{3}, \mathbf{4})$ is then exactly a matrix

$$
Y=\left(\begin{array}{llll}
y_{1,1} & y_{1,2} & y_{1,3} & y_{1,4} \\
y_{2,1} & y_{2,2} & y_{2,3} & y_{2,4} \\
y_{3,1} & y_{3,2} & y_{3,3} & y_{3,4}
\end{array}\right)
$$

satisfying the following constraints:

$$
\begin{aligned}
& y_{1,1}<y_{1,2}<y_{1,3}<y_{1,4} \\
& \begin{array}{c}
= \\
y_{2,1}
\end{array} \quad \stackrel{y_{2,2}}{=}<y_{2,3}<y_{2,4} \\
& =\quad=\quad \wedge \\
& y_{3,1}<y_{3,2}<y_{3,3}<y_{3,4}
\end{aligned}
$$

The generic equivalence classes for the equality relation are then equal to:

$$
\left\{y_{1,1}, y_{2,1}, y_{3,1}\right\},\left\{y_{1,2}, y_{2,2}, y_{3,2}\right\},\left\{y_{1,3}\right\},\left\{y_{2,3}, y_{3,3}\right\},\left\{y_{1,4}\right\},\left\{y_{2,4}\right\},\left\{y_{3,4}\right\}
$$

There is moreover a natural poset $P_{t}$ built on the generic equality equivalence classes of the matrix $Y$, whose partial ordering exactly is defined by the inequalities occuring in Conditions $\mathbf{H}$ and $\mathbf{V}(\mathbf{t})$. In the case of our previous example, the poset $P_{(3,4)}$ is for instance given by the graph (a) of Figure 1.

It is now immediate to see that the decomposition of $\Gamma_{t}(X)$ over the basis of quasi-monomial functions exactly is equivalent to find the set $\mathcal{Q}(t)$ of all weak-order linear quotients of $P_{t}$, i.e., of all total orders which are constructed over a set $\bar{Q}$ of the form $\bar{Q}=\left\{Q_{1}<Q_{2}<\ldots<Q_{q}\right\}$, where $\left(Q_{p}\right)_{1 \leq p \leq q}$ denotes a partition of the set of all entries of $Y$ such that each $Q_{i}$ is either an element of $P_{t}$ or the union of several elements of $P_{t}$, in such a way that the ordering of $P_{t}$ is always preserved. One can then check that

$$
\Gamma_{t}(X)=\sum_{\bar{Q} \in \mathcal{Q}(t)} M_{C(\bar{Q})}(X)
$$

where one sets $C(\bar{Q})=\left(c_{1}, c_{2}, \ldots, c_{q}\right)$, where $c_{p}$ stands itself, for every $1 \leq p \leq q$, for:

$$
c_{p}=\sum_{y_{k, l} \in Q_{p}} i_{k} j_{l} .
$$




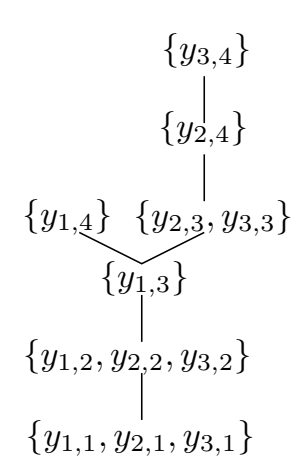

(a)

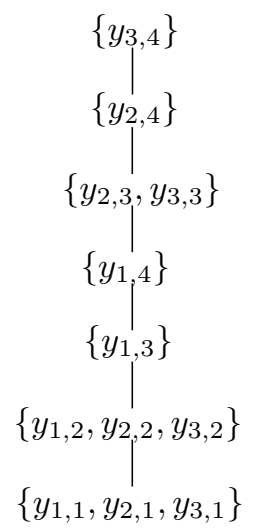

( e )

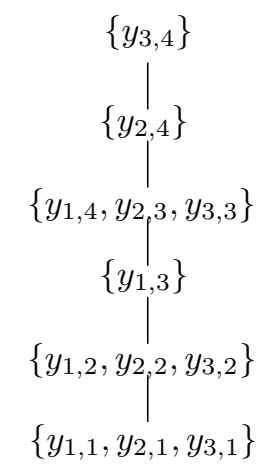

( $b$ )

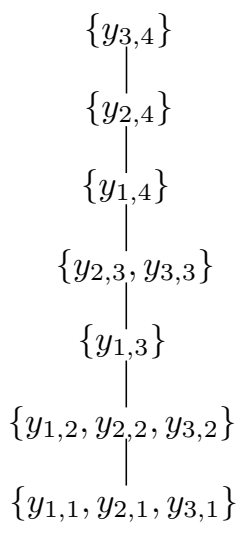

( $f$ )

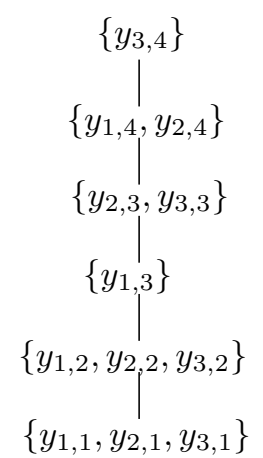

(c)

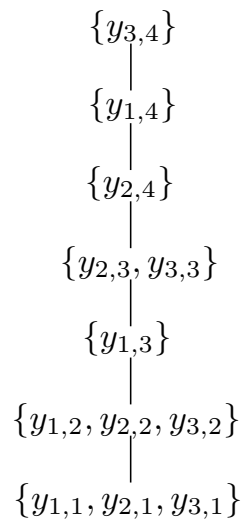

( g )

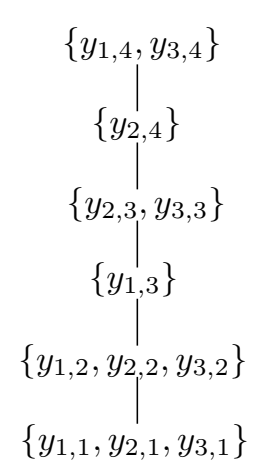

(d)

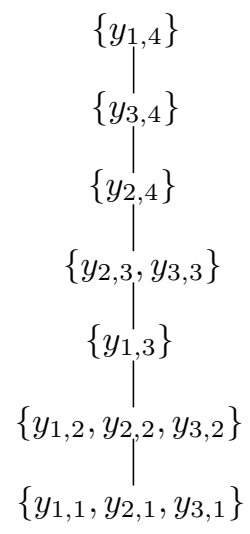

( h )

Figure 1: The poset $P_{(3,4)}((\mathrm{a}))$ and the weak-order extensions of $\mathcal{Q}((3,4))((\mathrm{b})-(\mathrm{h}))$.

Example 5.2 Let us again continue the previous example. The expansion of the quasi-symmetric function $\Gamma_{(3,4)}(X)$ on the basis of quasi-monomial functions can be immediately obtained from Figure 1 where one represented (from (b) to (h)) all the weak-order linear quotients of $P_{(3,4)}$ that compose $\mathcal{Q}(3,4)$. The compositions which are associated by formula (20) with all the weakorder linear quotients of $\mathcal{Q}(3,4)$ given in Figure 1 , are for instance listed below:

$$
\begin{array}{ll}
C(b)=(30,60,18,96,40,56), & C(c)=(30,60,18,72,64,56), \\
C(d)=(30,60,18,72,40,80), & C(e)=(30,60,18,24,72,40,56), \\
C(f)=(30,60,18,72,24,40,56), & C(g)=(30,60,18,72,40,24,56), \\
C(h)=(30,60,18,72,40,56,24) . &
\end{array}
$$

A simple application of Formula (19) then gives the expression of $\Gamma_{(3,4)}(X)$ on the basis of quasi-monomial functions:

$$
\begin{aligned}
\Gamma_{(3,4)}(X)= & M_{(30,60,18,96,40,56)}(X)+M_{(30,60,18,72,64,56)}(X)+M_{(30,60,18,72,40,80)}(X) \\
& +M_{(30,60,18,24,72,40,56)}(X)+M_{(30,60,18,72,24,40,56)}(X) \\
& +M_{(30,60,18,72,40,24,56)}(X)+M_{(30,60,18,72,40,56,24)}(X)
\end{aligned}
$$


It then comes that Formulas (18) and (19) reduce the computation of $M_{I}(X) \circ M_{J}(X)$ to the respective computations of

1. the poset $P_{t}$,

2. the set $\mathcal{Q}(t)$ of its weak-order linear quotients,

for every vector $t \in T$. The only non-trivial problem is now to be able to compute efficiently the set $\mathcal{Q}(t)$. Due to the fact that the poset $P_{t}$ is clearly a graded tree, all the weak-order linear quotients of $P_{t}$ can be computed using a slight adaptation, which is given below, of the general algorithm that computes all the weak-order extensions of a given poset (see [2]).

\section{function WEAK ;}

Input: $N$ : a set of minimal elements of $P_{t}$. $h$ : height of a maximal antichain.

Output: $C$ : all weak-order linear quotients of $P_{t}$.

\section{begin}

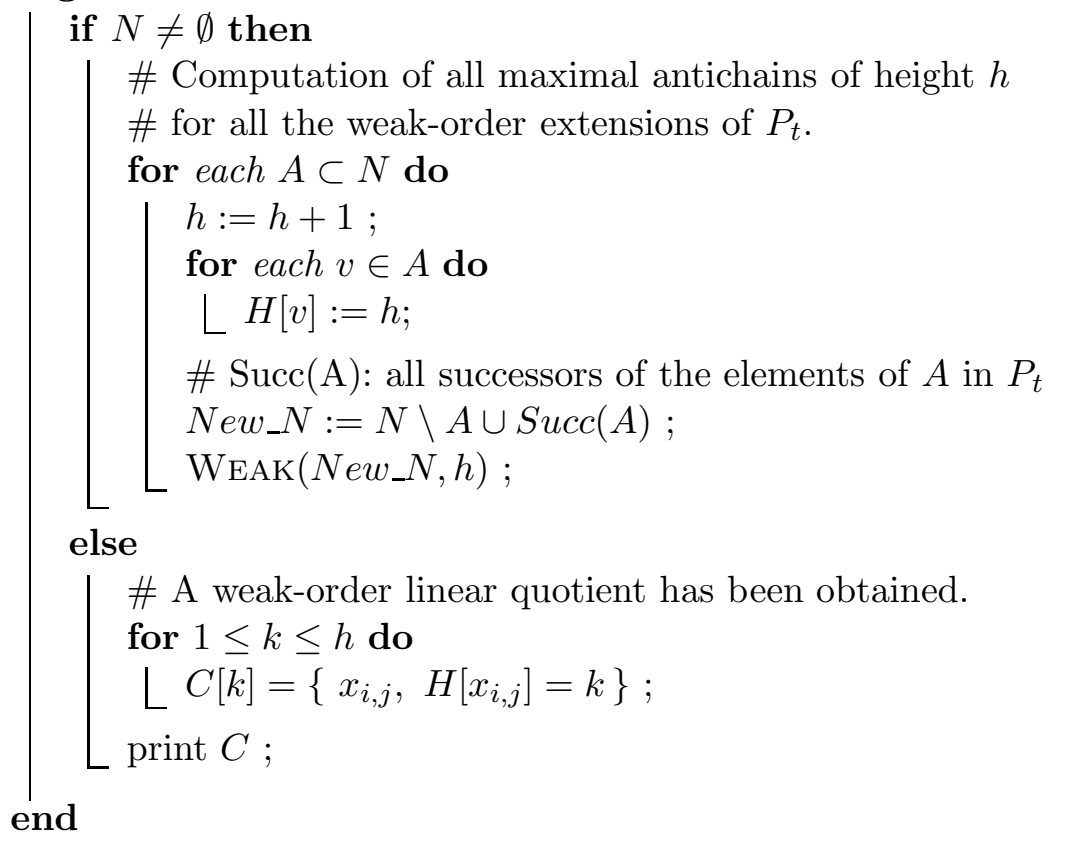

Algorithm 1: Function WEAK - Computation of all weak-order linear quotients of a poset.

The results of [2] indeed show that the function WEAK computes all weak-order linear quotients of $P_{t}$ when initialized with $h=0$ and $N$ equal to the generic equality equivalence class of $x_{1,1}$, i.e., with the set

$$
N=\left\{x_{i, j}, x_{i, j}=x_{1,1}\right\}
$$

Note 5.3 The reader will easily check that the complexity of our method for computing the plethysm $M_{I}(X) \circ M_{J}(X)$ as a sum of quasi-monomials functions is in time $O(m n p(I, J))$ and in space $O\left(n^{m-1}+m n^{2}\right)$, where $p(I, J)$ is the number of quasi-monomial functions involved in the expansion of the computed plethysm. 


\subsection{Plethysm of quasi-ribbon functions}

Let again $X$ be a totally ordered infinite set and let $I, J$ be two compositions. Let us first recall that Malvenuto and Reutenauer already proposed an algorithm for decomposing the plethysm of two quasi-ribbon functions on the basis of quasi-ribbon functions (see Proposition 4.9). We will now see how to improve the efficiency of their method.

Let $I=\left(i_{1}, \ldots, i_{r}\right)$ and $J=\left(j_{1}, \ldots, j_{s}\right)$ be respectively two compositions of $n$ and $m^{1}$. We will first recall that, by definition, one has:

$$
F_{J}(X)=\sum_{\left(y_{1}, \ldots, y_{m}\right) \in \mathcal{F}_{J}} y_{1} \ldots y_{m}
$$

where the sum is taken over the set $\mathcal{F}_{J}$ of all vectors $\left(y_{1}, \ldots, y_{m}\right)$ of $X^{m}$ satisfying Condition $C_{J}$ given below:

- Condition $\mathbf{C}_{\mathbf{J}}$ : one has

$$
\begin{cases}y_{k}<y_{k+1} & \text { for every } k \in D(I) \\ y_{k} \leq y_{k+1} & \text { for every } k \notin D(I)\end{cases}
$$

The commutative monomials that occur in the right hand side of the previous expression of $F_{J}(X)$ can be totally ordered by lexicographic order. Thus, according to the definition of the plethysm of two quasi-symmetric functions given in Section 4.2, we have:

$$
\begin{aligned}
F_{I}(X) \circ F_{J}(X) & =F_{I}\left(\left\{y_{1} \ldots y_{m},\left(y_{1}, \ldots, y_{m}\right) \in \mathcal{F}_{J}\right\}\right) \\
& =\sum_{\left(y_{1,1}, \ldots, y_{1, m}\right) \prec \prec_{1} \cdots \prec_{n-1}\left(y_{n, 1}<\cdots<y_{n, m}\right)} \prod_{\substack{i \in[1, n] \\
j \in[1, m]}} y_{i, j}
\end{aligned}
$$

where $\prec_{k}$ stands for $\preceq$ (the strict lexicographic order on $X^{m}$ ) when $k \in D(I)$ and for $\prec$ (the large lexicographic order on $X^{m}$ ) when $k \notin D(I)$. In other words, one has:

$$
F_{I}(X) \circ F_{J}(X)=\sum_{Y \in \mathcal{G}_{I, J}} \prod_{\substack{i \in[1, n], j \in[1, m]}} y_{i, j}
$$

where the above sum is taken over the set $\mathcal{G}_{I, J}$ of all $m \times n$ matrices

$$
Y=\left(\begin{array}{ccc}
y_{1,1} & \cdots & y_{1, m} \\
\vdots & \vdots & \vdots \\
y_{n, 1} & \cdots & y_{n, m}
\end{array}\right)
$$

over the alphabet $X$, satisfying the two conditions:

- Condition $\mathbf{H}_{\mathbf{J}}$ : for every $k \in[1, n]$, the vector $\left(y_{k, 1}, \ldots, y_{k, m}\right)$ satisfies Condition $\mathbf{C}_{\mathbf{J}}$;

- Condition $\mathbf{V}_{\mathbf{I}}$ : one has

$$
\left\{\begin{aligned}
\left(y_{k, 1}, \ldots, y_{k, m}\right) \prec\left(y_{k+1,1}, \ldots, y_{k+1, m}\right) & \text { for every } k \in\left\{i_{1}, i_{1}+i_{2}, \ldots, i_{1}+\ldots i_{r-1}\right\} \\
\left(y_{k, 1}, \ldots, y_{k, m}\right) \preceq\left(y_{k+1,1}, \ldots, y_{k+1, m}\right) & \text { for every } k \notin\left\{i_{1}, i_{1}+i_{2}, \ldots, i_{1}+\ldots i_{r-1}\right\} .
\end{aligned}\right.
$$

\footnotetext{
${ }^{1}$ Notice that $n$ and $m$ are not defined as in the previous section, since they do not play the same role if one considers the quasi-monomial functions or the quasi-ribbon functions.
} 
Let us now first recall the method used by Malvenuto and Reutenauer (see [14]) to prove the formula for the plethysm $F_{I}(X) \circ F_{J}(X)$ with formula (23). It is based on the following lemma that shows that Condition $\mathbf{V}_{\mathbf{I}}$ can be decomposed into a disjoint union of simple conditions.

Lemma 5.4 (Malvenuto, Reutenauer; [14]) There exist a set $\mathcal{R}$ of $2^{(n-1) m}$ matrices of the form $R \in \mathcal{M}_{n-1, m}$ where each $R_{i, j}$ is one of the inequalities $\{<, \leq,>, \geq\}$, such that a matrix $Y$ of $\mathcal{M}_{n, m}(X)$ satisfies Condition $\mathbf{V}_{\mathbf{I}}$ if and only if it satisfies one of the conditions $\mathbf{V}_{\mathbf{R}}$ defined by setting:

- Condition $\mathbf{V}_{\mathbf{R}}: \forall i \in[1, n-1], \forall j \in[1, m], y_{i, j} R_{i, j} y_{i+1, j}$.

The idea of the proof of this lemma is based on the following equivalences:

$$
\begin{aligned}
& (a, b) \prec\left(a^{\prime}, b^{\prime}\right) \Leftrightarrow\left\{\begin{array}{c}
a<a^{\prime} \text { and } b \geq b^{\prime} \\
a \leq a^{\prime} \text { and } b<b^{\prime}
\end{array}\right. \\
& (a, b) \preceq\left(a^{\prime}, b^{\prime}\right) \Leftrightarrow\left\{\begin{array}{c}
a \leq a^{\prime} \text { and } b \leq b^{\prime} \\
a<a^{\prime} \text { and } b>b^{\prime}
\end{array}\right.
\end{aligned}
$$

that can easily be extended by induction to higher orders, considering all the entries of a vector but the first one as playing the role of $b$ in the previous formulas. For instance, one can see that

$$
(a, b, c) \prec\left(a^{\prime}, b^{\prime}, c^{\prime}\right) \Leftrightarrow\left\{\begin{array}{lll}
a<a^{\prime} & \text { and } & (b, c) \geq\left(b^{\prime}, c^{\prime}\right) \\
a \leq a^{\prime} & \text { and } & (b, c)<\left(b^{\prime}, c^{\prime}\right)
\end{array}\right.
$$

Note 5.5 Notice that if one builds the elements of $\mathcal{R}$ as seen previously, a matrix is generally very similar to the previous one in the list. The main idea of our algorithm for computing the plethysm of to quasi-ribbon functions is to take into account this property: the algorithm we provide builds the so-called ideal lattice of a poset associated with the matrix by reusing as much as possible of the ideal lattice of the previous matrix in the list.

Let $R$ be an element of $\mathcal{R}$. Let us define

$$
\Delta_{R}(X)=\sum_{Y \in \mathcal{V}_{R} \cap \mathcal{H}} \prod_{\substack{i \in[1, n] \\ j \in[1, m]}} y_{i, j}
$$

where $\mathcal{V}_{R}$ (resp. $\mathcal{H}$ ) denotes the set of the matrices of $\mathcal{M}_{n, m}(X)$ satisfying Condition $\mathbf{V}_{\mathbf{R}}$ (resp. Condition $\mathbf{H}_{\mathbf{J}}$ ). It is then immediate to see that one has:

$$
F_{I}(X) \circ F_{J}(X)=\sum_{R \in \mathcal{R}} \Delta_{R}(X)
$$

which reduces the problem of computing the plethysm $F_{I}(X) \circ F_{J}(X)$ to the problem of computing $\Delta_{R}(X)$ for each $R \in \mathcal{R}$.

One can then compute $\Delta_{R}(X)$ as a sum of quasi-ribbon functions by using the technique invented by Malvenuto and Reutenauer in [14]. Let $R$ be an element of $\mathcal{R}$. One first associates with $R$ the two posets $P_{R}$ and $P_{R}^{\prime}$ built on $\bar{Y}=\left\{y_{i, j}, i \in[1, n], j \in[1, m]\right\}$ and defined by the relations 


$$
\begin{array}{ll}
x<_{P_{R}} y & \text { if } x \leq y \text { or } x<y, \\
x<_{P_{R}^{\prime}} y & \text { if } x \leq y \text { or } y<x .
\end{array}
$$

According to Proposition 3.5, that can easily be adapted to our context, we can deduce from Formula (25) that the quasi-ribbon functions that are involved in the decomposition of $\Delta_{R}(X)$, are in direct correspondance with the linear extensions of the poset $P_{R}$. This old result of Gessel immediately shows that the quasi-symmetric function $\Delta_{R}(X)$ is equal to

$$
\Delta_{R}(X)=\sum_{L \in L\left(P_{R}\right)} F_{C(L)}
$$

where, for each total order $L=\left\{l_{1}<\ldots<l_{n m}\right\}$ of $L\left(P_{R}\right)$, the composition $C(L)=\left(c_{1}, \ldots, c_{r}\right)$ of $n m$ is defined by asking that its associated descent set is equal to

$$
D(C(L))=\left\{i \in[1, n m-1], l_{i}>l_{i+1} \text { in } P_{R}^{\prime}\right\},
$$

where the defining inequality of this last set holds in $X$. The decomposition of $F_{I}(X) \circ F_{J}(X)$ over the basis of quasi-ribbon functions is then obtained by the conjunction of this last formula with relation $(26)$.

Example 5.6 Let us set $I=(1,1,1)$ and $J=(2,1)$. In this case, the computation of the plethysm $F_{(1,1,1)}(X) \circ F_{(2,1)}(X)$ is based on the study of the families of $3 \times 3$ matrices

$$
Y=\left(\begin{array}{lll}
y_{1,1} & y_{1,2} & y_{1,3} \\
y_{2,1} & y_{2,2} & y_{2,3} \\
y_{3,1} & y_{3,2} & y_{3,3}
\end{array}\right)
$$

satisfying Condition $\mathbf{H}_{(\mathbf{2}, \mathbf{1})}$ and Condition $\mathbf{V}_{(\mathbf{1}, \mathbf{1}, \mathbf{1})}$. This last condition exactly means that each row vector of $Y$ must be strictly smaller (in the lexicographic order) than its successor in $Y$. The family $\mathcal{R}$, given by Lemma 5.4 , consists in $2^{2.3}=64$ different matrices $R$.

Among all elements of $\mathcal{R}$, let us consider the relation $R$ given by

$$
\left(\begin{array}{lll}
\wedge \mid & \wedge & \vee \mid \\
\wedge \mid & \wedge & \vee \mid
\end{array}\right)
$$

So the complete set of inequalities that $Y$ has to satisfy can be represented as

$$
\begin{gathered}
y_{1,1} \leq y_{1,2}<y_{1,3} \\
\wedge \mid \\
y_{2,1} \leq y_{2,2}<y_{2,3} \\
\wedge \mid \\
y_{3,1} \leq y_{3,2}<y_{3,3}
\end{gathered}
$$

Let us now describe the computation of $\Delta_{R}(X)$, which represents the contribution of $R$ in the decomposition of the plethysm $F_{(1,1,1)}(X) \circ F_{(2,1)}(X)$ given by formula (26). To this aim, let us first write down the inequalities corresponding to the poset $P_{R}^{\prime}$ obtained by reverting all strict edges of the previous set: 


$$
\begin{gathered}
y_{1,1} \leq y_{1,2}>y_{1,3} \\
\wedge \mid \\
y_{2,1} \leq y_{2,2}>y_{2,3} \\
\wedge \mid \\
y_{3,1} \leq y_{3,2}>y_{3,3}
\end{gathered}
$$

One can then easily compute the two posets $P_{R}$ and $P_{R}^{\prime}$ associated with $R$. Both posets are represented in Figure 2.

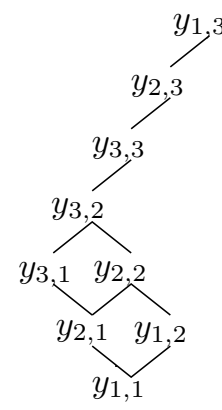

( a )

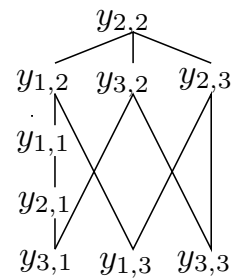

( b )

Figure 2: The posets $P_{R}$ and $P_{R}^{\prime}$ associated with $R$.

We can now compute for all linear extensions $L$ of $P_{R}$ the associated compositions $C(L)$ :

- $L=y_{1,1} \leq y_{2,1} \leq y_{3,1} \leq y_{1,2}<y_{2,2}<y_{3,2}<y_{3,3} \leq y_{2,3} \leq y_{1,3} \Rightarrow C(L)=(5,1,3)$

- $L=y_{1,1} \leq y_{2,1} \leq y_{1,2}<y_{3,1} \leq y_{2,2}<y_{3,2}<y_{3,3} \leq y_{2,3} \leq y_{1,3} \Rightarrow C(L)=(3,2,1,3)$

- $L=y_{1,1} \leq y_{2,1} \leq y_{1,2}<y_{2,2}<y_{3,1} \leq y_{3,2}<y_{3,3} \leq y_{2,3} \leq y_{1,3} \Rightarrow C(L)=(4,2,3)$

- $L=y_{1,1} \leq y_{1,2}<y_{2,1} \leq y_{3,1} \leq y_{2,2}<y_{3,2}<y_{3,3} \leq y_{2,3} \leq y_{1,3} \Rightarrow C(L)=(2,3,1,3)$

- $L=y_{1,1} \leq y_{1,2}<y_{2,1} \leq y_{2,2}<y_{3,1} \leq y_{3,2}<y_{3,3} \leq y_{2,3} \leq y_{1,3} \Rightarrow C(L)=(2,2,2,3)$

From these last considerations, it is now immediate to deduce the decomposition of $\Delta_{R}$ on the basis of quasi-ribbon functions:

$$
\Delta_{R}(X)=F_{(5,1,3)}(X)+F_{(3,2,1,3)}(X)+F_{(4,2,3)}(X)+F_{(2,3,1,3)}(X)+F_{(2,2,2,3)}(X) .
$$

Up to now, we presented the ideas of Malvenuto and Reutenauer in [14] to decompose the plethysm $F_{I}(X) \circ F_{J}(X)$ on the basis of quasi-ribbon functions. Their ideas are however not very effective and we will now present a technique to compute the plethysm that is based on the two following results of lattice theory.

An ideal of a poset $P$ is a subset $I$ of $P$ such that, for all $x \in I$, the relation $y \leq x$ implies that $y \in I$. The ideal lattice of $P$ is the set of all ideals of $P$ ordered by inclusion. This lattice is denoted by $I(P)=(I(P), U)$, where $U$ represents the cover relation.

Theorem 5.7 (Bonnet, Pouzet; [3]) There is a bijection between all linear extensions of a poset and the maximal chains of the ideal lattice of this poset. 
A result proved by Nourine (see $[15,7]$ ) can be transformed in a constructive way when used in conjunction ideas of interval doubling (see [1]) we will not present there. However, one finally gets to the following proposition:

Proposition 5.8 Let $P$ and $P^{\prime}$ be two posets such that $P^{\prime}=P \cup\{x\}$ where $x$ is a maximal element of $P^{\prime}$. Then the ideal lattice $T^{\prime}$ of $P^{\prime}$ can be constructed from the ideal lattice $T$ of $P$ by simply creating a copy of the interval $\left[x^{\prime}, \max (T)\right]$ in order to construct $\left[x, \max \left(T^{\prime}\right)\right]$ (where $x^{\prime}$ denotes the supremum of the set $A \subset P$ that consists of all predecessors of $x$ in $\left.P^{\prime}\right)$.

The main idea of our algorithm consists in treating the matrices $R$ column by column to use the fact that a matrix is generally very similar to the previous one in the list of $\mathcal{R}$. In order to present our algorithm, let us first introduce some new notations. For every $1 \leq v \leq m$, let us set

$$
Y_{v}=\left(\begin{array}{c}
y_{1, v} \\
\vdots \\
y_{n, v}
\end{array}\right) \quad \text { and } \quad Y_{1 \ldots v}=\left(\begin{array}{ccc}
y_{1,1} & \ldots & y_{1, v} \\
\vdots & \vdots & \vdots \\
y_{n, 1} & \ldots & y_{n, v}
\end{array}\right)
$$

Denote by $P_{v}$ and $P_{v}^{\prime}$ the posets corresponding to $Y_{v}$ and by by $T_{v}$ the ideal lattice of $P_{v}$. Denote by $L_{v}$ (resp. $L_{v}^{\prime}$ ) a linear extension of $P_{v}$ (resp. $P_{v}^{\prime}$ ). One defines in the same way $P_{1 \ldots v}$, $P_{1 \ldots v}^{\prime}, L_{1 \ldots v}, L_{1 \ldots v}^{\prime}$ and $T_{1 \ldots v}$ associated with the matrix $Y_{1 \ldots v}$.

Our algorithm consists in repeating the following operations when $R$ runs over the set $\mathcal{R}$ (we denote by $R^{\prime}$ the predecessor of $R$ in $\mathcal{R}$ if it exists):

1. Compute the greatest integer $v$ such that the first $v$-th columns of $R$ are the same as the first $v$-th columns of $R^{\prime}$.

2. Compute the ideal lattice of $Y_{1 \ldots v}$ by deleting in the ideal lattice of $R^{\prime}$ the elements $y_{k, l}$ with $l>v$.

3. Compute the ideal lattice $I\left(P_{R}\right)$ by adding each column one at a time as described in Proposition 5.8.

4. Compute $L_{R}$ and finally compute $\Delta_{R}(X)$.

Note 5.9 Let $I$ and $J$ be two compositions of respectively $n$ and $m$. The reader may easily check that our method for computing the plethysm $F_{I}(X) \circ F_{J}(X)$ has a time complexity of $O\left(2^{m n}\left(n m^{2}+|E|\right)\right)$ and a space complexity of $O(|E|+|X|)$, where $T=(X, E)$ is the ideal lattice of maximal size within all possible ideal lattices corresponding to a poset associated with a given choice of the graph $G_{R}$.

\section{References}

[1] Bertet K., Caspard N., Doubling convex sets in lattices: characterizations and recognition algorithms, Technical Report, LIAFA, Paris, France, 1999.

[2] Bertet K., Morvan M., Gustedt J., Weak-order extensions of an order, WG'97 (Möehring R.H., Ed.), Lectures Notes in Computer Science, Vol. 1335, 65-77, 1997.

[3] Bonnet R., Pouzet M., Extensions et stratifications d'ensembles dispersés, C. R. Acad. Sci. Paris, Série I, 1512-1515, 1969. 
[4] Duchamp G., Klyachko A., Krob D., Thibon J.Y., Noncommutative symmetric functions III : Deformations of Cauchy and convolution algebras, Disc. Math. and Theor. Comput. Sci., 1, 159-216, 1997.

[5] Gelfand I.M., Krob D., Leclerc B., Lascoux A., Retakh V.S., Thibon J.Y., Noncommutative symmetric functions, Adv. in Maths., 112, 218-348, 1995.

[6] Gessel I., Multipartitate P-partitions and inner products of skew Schur functions, [in "Combinatorics and algebra", C. Greene, Ed.], Contemporary Mathematics, 34, 289-301, 1984.

[7] Habib M., Medina R., Nourine L., Steiner G., A survey on generating ideals for posets and applications, Technical Research Report 95-033, LIRMM, Montpellier, France, July 1995.

[8] Knutson D., $\Lambda$-Rings and the Representation Theory of the Symmetric Group, Lect. Notes in Math., 308, Springer, 1973.

[9] Krob D., Leclerc B., Thibon J.Y., Noncommutative symmetric functions II : Transformations of alphabets, Int. J. of Alg. and Comput., 7, 181-264, 1997

[10] Krob D., Thibon J.Y., Noncommutative symmetric functions IV : Quantum linear groups and Hecke algebras at $q=0$, J. of Alg. Comb., 6, 339-376, 1997.

[11] Krob D., Thibon J.Y., Noncommutative symmetric functions $V:$ A crystalizable version of $U_{q}\left(G L_{n}\right)$, Int. J. of Alg. and Comput. (to appear).

[12] Lascoux A., Schützenberger M.P., Formulaire raisonné de fonctions symétriques, Université Paris 7, 1985.

[13] Malvenuto C., Reutenauer C., Duality between quasi-symmetric functions and Solomon descent algebra, J. Algebra, 177, 967-982, 1995.

[14] Malvenuto C., Reutenauer C., Plethysm and conjugation of quasi-symmetric functions, Preprint, Montréal, 1995.

[15] Nourine L., Quelques propriétés algorithmiques des treillis, PhD Thesis, Université de Montpellier, France, July 1995.

[16] Stanley R., Ordered structures and partitions, Mem. Amer. Math. Soc., 119, 1972. 